\title{
The GABAA receptor and benzodiazepine acceptor site
}

\author{
Michel Bourin* \\ Neurobiology of anxiety and mood disorders, University of Nantes, France
}

Received: February 23, 2018; Accepted: March 14, 2018; Published: March 16, 2018

*Corresponding author: Michel Bourin, Neurobiology of anxiety and mood disorders, University of Nantes, 98, rue Joseph Blanchart, 44100 Nantes, France, E-mail: michel.bourin@univ-nantes.fr

\begin{abstract}
The GABAA receptor is a member of the ion channel receptor family. It is sensitive to muscimol (agonist) as well as bicuculline and picrotoxin (antagonists). The binding of GABA on its recognition site causes the opening of a chlorine ( $\mathrm{Cl}-$ ) channel, which, allowing the $\mathrm{Cl}$ - ions to pass, produces the hyperpolarization of the target cell. The GABAA receptor is a transmembrane glycoprotein composed of 4 subunits, alpha, beta, gamma and delta, currently recognized. It is sensitive to muscimol (agonist) as well as bicuculline and picrotoxin (antagonists). There are several types of GABAA receivers, different from each other by some of their subunits. There are currently 6 subtypes of alpha subunits, 3 subtypes of beta subunits, 3 subtypes of gamma subunits and 1 subtype of delta subunits. This entails not only a great heterogeneity of structure but also a pharmacological heterogeneity, the consequences of which are still poorly understood. The GABAA receptor has, besides the GABA receptor sites, a variety of other topographically distinct receptor sites capable of recognizing pharmacologically active substances, such as benzodiazepines (BZDs) - barbiturates - neurosteroids - convulsants - alcohol. These substances act in an allosteric manner with the GABA receptor sites and modulate the GABAA response.
\end{abstract}

Key words: Receptor GABAA; Benzodiazepines Site; Allostery; Channel Chlorine

\section{Introduction}

Benzodiazepines havevery homogeneous pharmacodynamics properties [1]; such similarity of action led to the discovery of a common neurobiochemical activity. Thus, the notion of acceptor site with benzodiazepines [2] was revealed according to the same mode of thought which had made it possible to discover the opioid receptors.

Most benzodiazepines (BZDs) are agonists that promote the opening of the Cl- channel by GABA and therefore have an inhibitory effect. They have common pharmacological properties: they are anxiolytic, hypnotic, anticonvulsant; muscle relaxant and can have an amnesic effect. As a result, they potentially have the same indications and the same side effects.

The purpose of this article is therefore to present a review of the GABAA receptor physiology and its benzodiazepine modulator site.

\section{Distribution of GABAA receptors in the brain}

\section{Anatomical location "in vitro"}

If the affinity of the receptors for diazepam is the same in all regions of the brain, the density is very variable. The strongest concentration is in the frontal cortex. These receptors exist at lower concentrations in the cerebellar cortex, hypothalamus, hippocampus, amygdala and striatum. The lowest density is at the marrow bridge. Finally, there is a total absence of receptors in the subcortical white matter. This unequal distribution of benzodiazepine receptors corresponds to the localization of GABA [3].

\section{Anatomical location "in vivo"}

The method consists in using the positron emission tomography technique. Flunitrazepam, labeled with carbon 11, is administered intravenously at doses corresponding to $15-30$ micromoles of cold flunitrazepam. A few minutes after in vivo administration of $11 \mathrm{C}$ flunitrazepam, the radioactivity is greater in the temporal, parietal and occipital cortex [4].

\section{Intracellular localization}

There is no re-uptake of diazepam inside nerve cells. This suggests that the benzodiazepine site of action is located on the cell surface rather than inside the cell itself. The receptors are on the other hand associated with synaptic membranes and control the opening of a chlorine channel. On the ontogenetic level, it has been demonstrated in rats that benzodiazepines are fixed very rapidly just after birth. This goes against the different neuromediators for which the receptors develop later. At the phylogenetic level, benzodiazepine receptors appear late in the evolution of species. Thus, invertebrates are lacking of receptors and amphibians have very few. Then we find the reptiles, the birds which have more. Finally, all mammals have benzodiazepine receptors [5].

\section{The different constituents of the GABAA complex}

\section{The GABA system}

The dominant function of gamma-aminobutyric acid is its inhibitory activity [6]. GABA is the first amino acid whose role in neurotransmission was recognized. The nervous system has 
a concentration of GABA 200 to 1,000 times higher than that of other neurotransmitters (acetylcholine, serotonin ...).

- In the spinal cord: the gray substance, in the anterior horn and in particular in the gelatinous substance of Rolando, contains GABAergic interneurons. This location may explain the muscle relaxant activity of benzodiazepines [7].

- In the cerebellum: the cerebellum contains very many cells of different structures and functions. GABA is the neuromodulator of inhibitory interneurons such as Golgi basket cells and star cells. These neurons send efferences to excitatory cells and fibers whose neuromodulators are aspartic and glutamic acids. Likewise, GABAergic Purkinje cells are the only neurons to send efferences out of the cerebellum to deep nuclei [8].

- In the extra-pyramidal system: in the striatum, there are also local short-acting regulating interneurons and a striato-nigger GABAergic pathway that inhibits dopaminergic pathway activity [9].

- Finally, in the cerebral cortex: there are many GABAergic interneuron circuits regulating cortical excitability. Such a physiological function explains, on the one hand, the current trials in the treatment of gabamimetic molecules in the treatment of certain forms of epilepsy and, on the other hand, the antiepileptic activity of benzodiazepines [10].

\section{Functioning of the GABAergic synapse}

GABA is probably not bound to vesicles and attempts to isolate it at the level of synaptosomes have been unsuccessful. It is possible, however, that GABA, easily releasable, is in free form in nerve endings. On the other hand, no degradation enzyme was found in the synapse. Gabatransaminase is essentially mitochondrial, intracellular. This specificity confirms the phylogenetic hypothesis of bacterial symbiotic integration [11].

The stimulation of the GABAergic neuron leads, as for all the neuromodulators, a massive release of the molecule in the synaptic space. The released GABA has a quadruple become:

1. It binds to the postsynaptic GABA receptor with cyclic AMP formation.

2. It also attaches to a presynaptic receptor. This structure has the role of regulating the release of GABA in the synapse. This is a negative feedback.

3. It tends to diffuse out of the synapse in large quantities and thus extend its inhibitory activity to other neurons in the environment.

4. Finally it is recaptured by the presynaptic neuron where it is degraded by mitochondrial GABA transaminase [12].

\section{Ionophore chlorine}

The physiology of nerve cells is governed by electrical and concentration gradients. There are differences in ionic concentrations on both sides of the membrane. The gradient thus obtained tends to make the $\mathrm{Na}+$ and $\mathrm{Cl}$ - ions enter and the $\mathrm{K}+$ ion to go out.
At the same time, the polarization of the cell membrane (positive outer surface, negative inner surface) tends to make the $\mathrm{Na}+$ and $\mathrm{K}+$ cations enter and the $\mathrm{Cl}$ - anion to leave. The resultant of these opposing forces creates a potential difference (D.D.P.) of $-70 \mathrm{mV}$ in the quiescent state.

GABA involves a second messenger, the cyclic AMP (13). The binding of the neuromodulator with its receptor activates an adenylcyclase attached to the inner wall of the membrane. This molecule transforms AMP into cyclic AMP. This newly synthesized molecule binds under the inhibitory subunit of a membrane protein kinase. There is then a dissociation of the protein kinase which releases a catalytic subunit. This subunit transfers a phosphate radical of ATP to a neighboring protein substrate. This phosphorylated substrate changes its conformation or its position and thus allows the opening of the ionophore chlorine.

\section{Primary structure of the receptor complex}

GABAA receptors belong to the family of membrane receptors associated with an ion channel. It is a complex formed of several subunits (it is a transmembrane glycoprotein hetero-oligomer).

Recently biochemical studies and molecular cloning studies have revealed the primary structure of the GABAA complex [14].

The first hypothesis considered was that of a complex formed of alpha and beta subunits. Determination of the molecular size of the receptor suggested a tetrameric complex. However, a pentameric structure was retained because of the open pore diameter $(0.56 \mathrm{~mA})$ (Figure 1$)$.

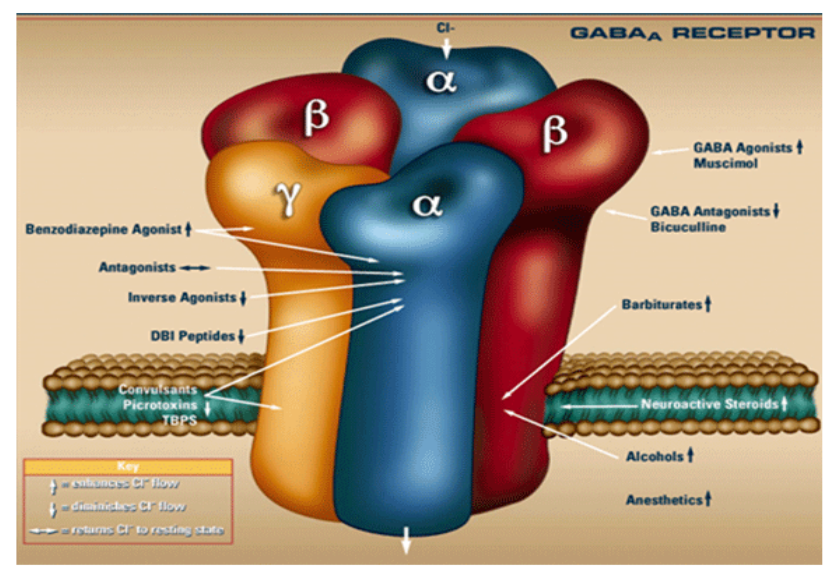

Figure 1: Scheme of GABAA receptor

GABA would bind to beta subunits and the benzodiazepine binding site would be localized at the alpha subunit level [15]. In vitro reconstitution of such a receptor may in some cases produce a weak effect of benzodiazepines on the activation of the chlorine channel by GABA [16]. Electrophysiological studies of oocytes have revealed that alpha and beta subunit receptors may form the GABA-bound receptor channel, and this receptor may be blocked by bicuculline and picrotoxin. But the combination of alpha and beta is not enough to explain the pharmacological response to benzodiazepines. A ternary combination that additionally 
includes a gamma subunit is necessary to produce a correct GABA response, as well as its potentiation by benzodiazepines as normally observed [17]. A receptor composed of subunits (alpha, beta, and gamma) best matches the native receptor, and the simultaneous presence of these 3 subunits provides the correct pharmacological effect. The current hypothesis is based on the cloning of 4 different subunits: (alpha, beta, gamma, and delta). These four subunits have less than $50 \%$ homologous sequences, and variations greater than $70 \%$. For example gamma2 at $40 \%$ sequence identical to alpha and beta. The structure of each subunit consists of a broad extracellular ( $\mathrm{N}$-terminal) domain, 4 transmembrane domains (M1 to M4), a cytoplasmic loop between M3 and M4 and a short extracellular C-terminal region. In addition there are different isoforms of each subunit. We identified: 6 alphas; 4 betas; 2 gammas and 1 delta. But neither the stoichiometry nor the number of copies of each isoform are currently known. The use of polyclonal antibodies has demonstrated that the alpha1 and gamma2 subunits are integral components of the GABA receptor. The distribution of the different isoforms of each subunit is not homogeneous. In the rat brain, the alpha1, beta2 and gamma2 forms are the most distributed, and the most abundant. Other subunits have a smaller or even unique distribution. Of all the subunits, alpha6 is the most restricted, it can play a role in the benzodiazepine tolerance [18].

\section{Mechanisms of GABA receptor modulation by its different ligands}

There is a broad spectrum of ligands capable of binding to the GABA complex (Table 1).

\begin{tabular}{|l|}
\hline Table 1: Ligands that can modulate the GABAA complex \\
\hline Benzodiazepines \\
\hline Beta carbolines (CCE beta) \\
\hline Cyclopyrrolones (zopiclone, suriclone) \\
\hline Imidazobenzodiazepinones (flumazenil) \\
\hline Imidazopyridine (alpidem, zolpidem) \\
\hline Imidazopyrimidine \\
\hline Pyrazoloquinolinone \\
\hline Pyrazolopyridine \\
\hline Triazolopyridazine \\
\hline
\end{tabular}

The receptor-ligand binding makes it possible to detail two properties of the ligand:

- Its affinity for the receptor

- Its intrinsic effectiveness.

Agonists, inverse agonists, and antagonists are distinguished. If an agonist or inverse agonist has low activity and low ability to activate the receptor, it is considered a partial agonist (as opposed to complete agonists). Complete agonists increase the response to GABA. Their intrinsic activity is positive (positive allostery) [19]. They modulate the receptor by increasing the ability of GABA to act on the chlorine channel.
Inverse agonists produce the opposite effect. Complete agonists (inverse or not) induce a maximal pharmacological effect often before all receptors are activated [20]. All the binding sites of the macromolecular complex are allosterically connected, so that binding on one subunit modifies binding kinetics on the other subunits.

Partial agonists (inverse or not) modulate the receptor in the same way, but with a lower activity. Antagonists have no intrinsic activity (Figure 2). The binding of two molecules of GABA on the receptor complex makes it possible to activate it and to allow the opening of the channel.

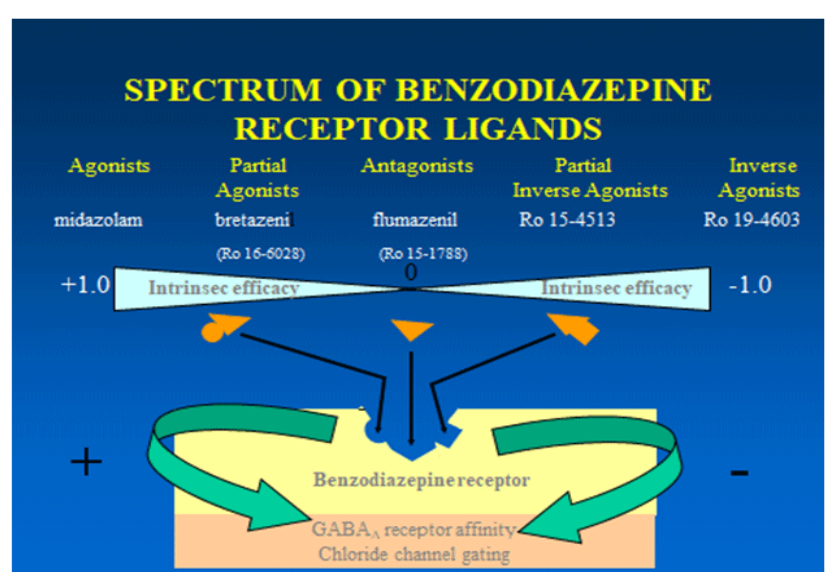

Figure 2: Spectrum of benzodiazepine receptor ligands

Benzodiazepine receptor agonists would stabilize the receptor in high affinity conformation, whereas inverse agonists would stabilize the receptor in a low affinity conformation. Partial agonists act similarly on both conformations, but to a lesser extent because it distinguishes the two states less well. Partial agonists possess only a part of the properties of complete agonists.

\section{Different classifications of the GABAA receptors and their modular sites}

Receptors in the central nervous system have been subdivided into two functional groups: high affinity receptors and low affinity receptors.

At rest the receptors are in a low affinity state and not occupied. When sufficient quanta of GABA are released, an increase in the $\mathrm{Kd}$ of the receptor occurs.

The presence of benzodiazepines would make the receptors sensitive to small amounts of GABA, which are insufficient outside this sensitization. The different isoforms of each unit could explain the existence of several types of GABAA receptors. The macro-molecular complexes formed of subunits (alpha, beta, gamma) would be sensitive to benzodiazepines and the complexes (alpha, beta, delta) would not be.

GABA receptors are subdivided according to the presence or absence of GABA modulin. Thus, the GABA2 receptor is associated with modulin, which depends on the allosteric structure of this high affinity receptor. In contrast, the low affinity GABA1 receptor 
is not regulated by modulin [21]. Currently, classification attempts are based on the type of each isoform present in the receptor. But not all combinations are possible in vivo; moreover, there are several hundred possible. Electrophysiological and pharmacological studies have shown that the properties of different receptors depend on the subunits present. A system therefore relies not only on the composition in subunits but also on the property of the receiver. The receivers are then subdivided into 5 classes (see Table 2). Given the current knowledge of the GABA receptor, a classification taking into account the alpha, beta, and gamma and non-delta subunits is sufficient.

\begin{tabular}{|l|}
\hline Table 2: GABAA Receptor Classification Model \\
\hline Type I: alpha1, beta2, gamma2 \\
\hline Type II: alpha2 or alpha3, beta2, gamma2 \\
\hline Type III: alpha5, beta3, gamma2 \\
\hline Type I: alpha6, beta2, gamma2 \\
\hline Type V: alpha1, beta1, gamma1 \\
\hline
\end{tabular}

There is another classification using omega nomenclature [22]. Indeed, a classification based solely on benzodiazepine receptors seems too restrictive. Compounds other than those belonging to the benzodiazepine class, imidazopyridines for example, thus bind to these same receptors with high affinity. Omega receptors would classify all chemical compounds, including benzodiazepines that bind to the same sites. Thus omega1 receptors are related to BZ1 receptors [23].

\section{Conclusion}

Molecular pharmacology has provided us with some keys to better understand the interactions that may exist between several drugs such as benzodiazepines, imidazopyridines and cyclopyrrolones, and barbiturates. It is easier to understand the links between convulsions, sedation, anxiolysis and myorelaxation since they are drugs that act at nearby sites of action that prove effective. A very practical conclusion, do not associate benzodiazepines and other drugs fixing on the macromolecular site, because they can compete (24).

\section{References}

1. Dailly E, Bourin M. The use of benzodiazepines in the aged patient: clinical and pharmacological considerations. Pak J Pharm Sci. 2008;21(2):144-150.

2. Renard S, Olivier A, Granger P, Avenet P, Graham D, Sevrin M, et al. Structural elements of the gamma-aminobutyric acid type A receptor conferring subtype selectivity for benzodiazepine site ligands. J Biol Chem. 1997;274(19):13370-13374.

3. Schoch P, Richards JG, Häring P, Takacs B, Stähli C, Staehelin T, et al. Colocalization of GABA receptors and benzodiazepine receptors in the brain shown by monoclonal antibodies. Nature. 1985;314(6007):168171.

4. Frey KA, Holthoff VA, Koeppe RA, Jewett DM, Kilbourn MR, Kuhl DE. Parametric in vivo imaging of benzodiazepine receptor distribution in human brain. Ann Neurol. 1991;30(5):663-672.
5. Enna SJ, Gallagher JP. Biochemical and electrophysiological characteristics of mammalian GABA receptors. Int Rev Neurobiol. 1983;24:181-212.

6. Capogna M, Pearce RA. GABA A, slow: causes and consequences. Trends Neurosci. 2011;34(2):101-112.

7. Price GW, Kelly JS, Bowery NG. The location of GABAB receptor binding sites in mammalian spinal cord. Synapse. 1987;1(6): 530-538.

8. Dizon MJ, Khodakhah K. The role of interneurons in shaping Purkinje cell responses in the cerebellar cortex. J Neurosci. 2011;31(129):10463-10473.

9. Momiyama T, Nishijo T. Dopamine and Serotonin-Induced Modulation of GABAergic and Glutamatergic Transmission in the Striatum and Basal Forebrai. Front Neuroanat. 2017;11:42. doi: 10.3389/ fnana.2017.00042

10. Möhler H, Fritschy JM, Crestani F, Hensch T, Rudolph U. Specific GABA (A) circuits in brain development and therapy. Biochem Pharmacol. 2004 ;68(8):1685-1690. DOI: 10.1016/j.bcp.2004.07.025

11.Guidotti A, Corda MG, Wise BC, Vaccarino F, Costa E. GABAergic synapses. Supramolecular organization and biochemical regulation. Neuropharmacology. 1983;22(12B):1471-1479.

12. Cherubini E, Conti F. Generating diversity at GABAergic synapses. Trends Neurosci. 2001;24(3):155-162.

13. Mehta AK, Ticku MK. Chronic GABA exposure down-regulates GABAbenzodiazepine receptor-ionophore complex in cultured cerebral cortical neurons. Brain Res Mol Brain Res. 1992;16(1-2):29-36.

14. Chua HC, Chebib M. GABAA Receptors and the Diversity in their Structure and Pharmacology. Adv Pharmacol. 2017;79:1-34. DOI: 10.1016/bs.apha.2017.03.003

15. Pálvölgyi A, Móricz K, Pataki Á, Mihalik B, Gigler G, Megyeri K, et al. Loop $F$ of the GABA A receptor alpha subunit governs GABA potency. Neuropharmacology. 2018;128:408-415. DOI: 10.1016/j. neuropharm.2017.10.042

16. Wongsamitkul N, Maldifassi MC, Simeone X, Baur R, Ernst M, Sigel E. $\alpha$ subunits in GABAA receptors are dispensable for GABA and diazepam action. Sci Rep. 2017;7(1):15498. doi:10.1038/s41598-017-15628-7

17. Uusi-Oukari M, Korpi ER. Regulation of GABA (A) receptor subunit expression by pharmacological agents. Pharmacol Rev. 2010;62(1):97135. DOI: $10.1124 /$ pr.109.002063

18. Leggio GM, Torrisi SA, Castorina A, Platania CB, Impellizzeri AA, Fidilio A, et al. Dopamine D3 receptor-dependent changes in alpha6 GABAA subunit expression in striatum modulate anxietylike behaviour: Responsiveness and tolerance to diazepam. Eur Neuropsychopharmacol. 2015;25(9):1427-1436 DOI: 10.1016/j. euroneuro.2014.11.004

19. Olsen RW. Allosteric ligands and their binding sites define $\gamma$-aminobutyric acid (GABA) type A receptor subtypes. Adv Pharmacol. 2015;73:167-202. DOI: 10.1016/bs.apha.2014.11.005

20. Atack JR. Preclinical and clinical pharmacology of the GABAA receptor alpha5 subtype-selective inverse agonist alpha5IA. Pharmacol Ther. 2010;125:11-26. DOI: 10.1016/j.pharmthera.2009.09.001

21. Beltrán González AN, Pomata PE, Goutman JD, Gasulla J, Chebib M, Calvo DJ. Benzodiazepine modulation of homomeric GABAA 1 receptors: differential effects of diazepam and 4'-chlorodiazepam. Eur J Pharmacol. 2014; 743:24-30. DOI: 10.1016/j.ejphar.2014.09.017 
22. Joksimović $S$, Varagic Z, Kovačević J, Van Linn M, Milić M, Rallapalli $S$ et al. Insights into functional pharmacology of $\alpha_{1}$ GABA (A) receptors: how much does partial activation at the benzodiazepine site matter? Psychopharmacology (Berl). 2013;230(1):113-123. DOI: 10.1007/ s00213-013-3143-4

23. Yakushiji T, Shirasaki T, Munakata M, Hirata A, Akaike N. Differential properties of type I and type II benzodiazepine receptors in mammalian CNS neurones. Br J Pharmacol. 1993;109(3):819-825.
24. Bourin M. Do benzodiazepines still need to be used? Open Access J Neurol Neurosurg. 2017 5(4):555669. DOI: 10.19080/ OAJNN.2017.05.5556670 